\title{
High Resolution Remote Sensing Observations at the Virginia Coastal Reserve Long-Tem Ecological Research Site
}

\author{
Timothy F. Donato, Charles M. Bachmann, and Robert A. Fusina \\ Naval Research Laboratory, Remote Sensing Division, Code 7210, Washington, DC 20375 \\ John H. Porter \\ Department of Environmental Sciences, University of Virginia, Charlottesville, VA 22903 \\ Barry Truitt \\ The Nature Conservancy, Virginia Coastal Reserve, P.O. Box 158, Nassawadox, VA 23413
}

\begin{abstract}
In this investigation, we use data from $\mathrm{HyMap}^{\top \mathrm{M}}$, an airborne hyperspectral sensor, and IKONOS, a space borne high spatial resolution multispectral sensor, to investigate the landscape structure and variability of a coastal tidal marshbarrier island complex. Specifically, we explore the relationship between hyperspectral signature and patch metrics, such as Core Area Index, Shape, and Square Pixel among others.
\end{abstract}

\section{INTRODUCTON}

The marriage between remote sensing and landscape ecology is a natural one. Landscape ecology in its broadest sense is defined as the study of the effect of landscape pattern on ecological processes [1]. Specifically, [2] express that landscape ecology considers the development and dynamics of spatial heterogeneity, interactions, and exchanges through landscape boundaries or ecotones, the influence of spatial heterogeneity on biotic and abiotic processes, and the management of the spatial heterogeneity. Quantitative methods are required to compare different landscapes, identify temporal fluctuations and shifts, and relate pattern to ecological process. These quantitative measures or metrics are discussed in detail in [1], [3], and [4].

In this study, we examine the spectral variability of emergent Spartina alterniflora patches in a coastal landscape using data collected from HyMap, an airborne hyperspectral sensor, and IKONOS, a space borne high spatial resolution multispectral sensor. Specifically we examine the spectral variability associated from each patch relative to the entire class observation. Employed metrics include, Core Area Index, Shape and Square Pixel, among others

\section{METHODS AND MATERIALS.}

\section{A. Study Area}

Extending $110 \mathrm{~km}$ along the seaward margin of the southern Delmarva Peninsula, the Virginia Coastal Reserve Long-Term Ecological Research (VCR-LTER) site provides the setting for this study. The VCR, characterized as a prototypical barrier island/lagoonal marsh/estuarine complex, consists of a complex assemblage of 14 barrier islands that is interlaced with a network of deep tidal channels, inlets, flats, and marshes. The high resolution data examined in this study was acquired over Smith Island, (Fig 1). Located at the southern end of the peninsula, Smith Island is characteristic of the barrier islands within the VCR. The island is approximately $11 \mathrm{~km}$ long, has an average height above mean-seal-level of only $1.5 \mathrm{~m}$, and is known to be, at times, bisected by ephemeral tidal inlets, the most recent inlet closing occurring around 1970 [5].

\section{B. Data and Methods}

Airborne hyperspectral data was acquire over Smith Island (Fig 1.) with the HyMap sensor on 8 May 2000 at 15:55 UTC. For this acquisition, HyMap was configured with a 128 bands ranging from approximately $450 \mathrm{~nm}$ to $2400 \mathrm{~nm}$ with a nominal $20 \mathrm{~nm}$ bandwidth. The delivered radiance product had a $4.5 \mathrm{~m}$ ground sampling distance or GSD. Atmospheric corrections were performed by the data provider using ATREM [6].

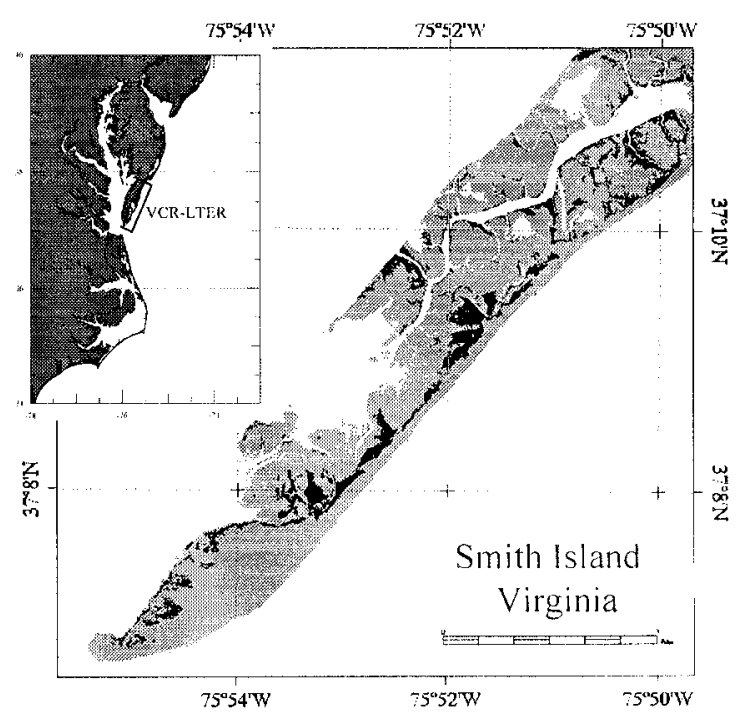

Fig. I Smith Island study area depicting the HyMap image swath (shaded region) and emergent smooth cordgrass (Spatina alterniflora) patches, denoted by black regions, used in the landscape analysis. The entire region correspondence to the area imaged by Ikonos

TFD, CMB, and RAF supported by ONR, through NRL from Program N0001400WX0016 and N0001401 WX40009 JHP supported by NSF grants BSR-8702333-06, DEB-9211772, DEB-9411974, and DEB-0080381 
High resolution multispectral data was acquired over Smith Island on 6 June 2000 from the Ikonos satellite. The data set contains a georectified panchromatic channel with a GSD of $1 \mathrm{~m}$ (Fig 2.) and four multispectral channels, each with a GSD of $4 \mathrm{~m}$. The first four channels are similar in character to the Thematic Mapper carried on board recent Landsat satellites.

The following steps were performed on the hyperspectral data before the landscape analysis. We classified the scene by iirst performing a Maximum Noise Fraction (MNF) transform [7] on the data. We kept the first $20 \mathrm{MNF}$ transformed images and discarded the remainder. We then inspected the remaining transformed images for spectral, spatial, and textural qualities that would enhance the classification of the regions containing $S$. alterniflora. MNF bands 3, 4, and 6 proved optimal. Finally, we performed an unsupervised ISODATA classification [7] on the selected bands. A binary mask emphasizing $S$. alterniflora patches was subsequently generated from the classified result. This mask was used as the input scene for FRAGSTATS, a program that quantifies landscape structure (brevity prohibits a further discussion, and so we refer the reader to [4]). Several patch metrics where computed including the ones used in the analysis: Area, Perimeter Length, Core Area, Shape, Fractal Dimension, Number of Core Areas per Patch and Core Area Index (CAI). In addition, a Square-Pixel (SqP) metric [8] was computed.

One component of the FRAGSTATS output is a patch identification mask. This mask was used as a template for generating basic spectral statistics for each patch and the entire $S$. alterniflora class. The mean class spectrum for $S$. alterniflora was then used as the reference for Spectral Feature Fitting (SFF). The SFF method is discussed in [9] and implemented in ENVI. SFF reports scores based on spectral similarity confidence. High scores indicate a strong similarity between the observed and reference spectrum. In our case, a high score indicates a strong similarity between the mean patch spectrum and the mean class spectrum.

\section{OBSERVATION AND RESULTS}

The mask generated from the classified MNF result is depicted in Fig 1 and is denoted by the black highlighted areas. These areas are associated with $S$ alterniflora communities that occupy slightly elevated regions. A Total of 248 patches were identified by FRAGSTATS. The patches ranged in area from 0.1 to 31 ha with an average of 0.89 ha and a standard deviation of 2.97 ha. Fifty percent of the patches are less than 0.2 ha (Note: 1 ha $=10,000 \mathrm{~m}^{2}$ ). Fig. 3 a depicts the patch area distribution.

The mean shape for the observed patches was 2.8 and over $70 \%$ of the reported values were under 2.5 , which indicates a modest amount of complexity in regards to patch shape. The Shape Index and Square Pixel are area-perimeter metrics that measure patch shape complexity with respect to a standard square pixel. The Shape Index histogram is shown in Fig3b.

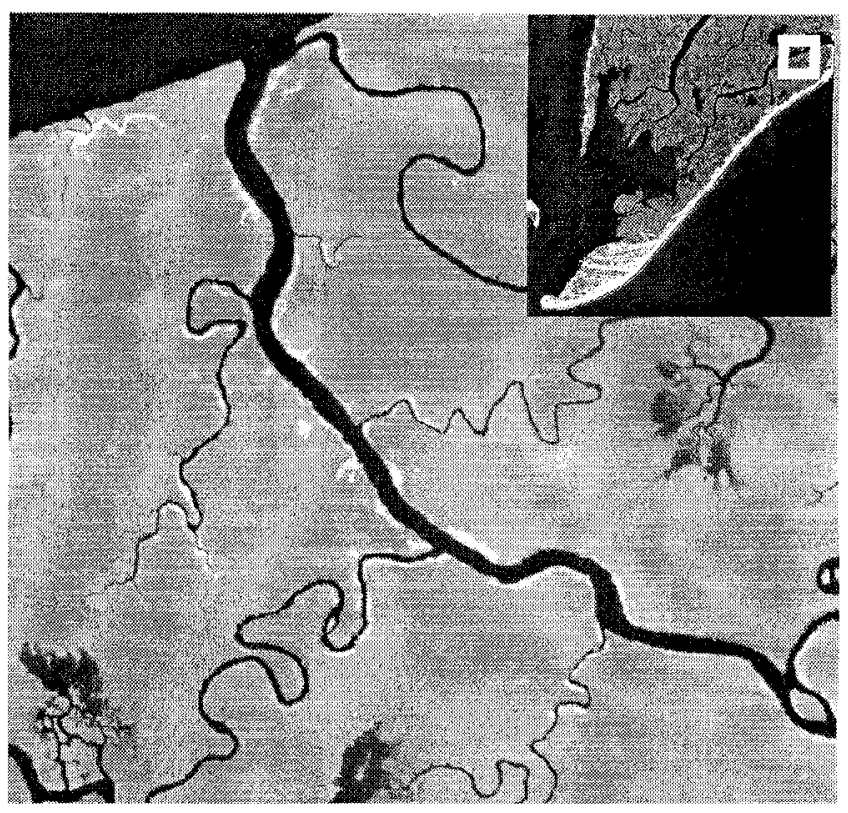

Fig. 2 A subset from the Ikonos $1 \mathrm{~m}$ panchromatic channel. Inset dipicts the actual inage area. The white box denotes the location of the depicted sample. Sublte changes in gray scale intensity denote relief and regions of inundation. (Ikonos imagery (C2000 Space imaging)

The Core Area Index represents the percentage of patch that is unaffected by edge and is basically an edge-to-interior ratio. The mean CAI is about 43 and the metric is more normally distributed than the two previously report results (Fig. 3c).

SFF results are present Fig. $3 \mathrm{~d}$ and reveal that over $50 \%$ of the individual patch spectra are within $15 \%$ of the reference. This result indicates a fairly homogenous patch structure with respect to the entire class of observations and it further implies a spectral homogeneity regarding $S$. alterniflora community structure.

\section{DISCUSSION}

The HyMap data were collected approximately one hour before high slack water, so much of the marsh was inundated. In addition, the data were collected early in the growing season, before the full extent of growth was observable. Both matters complicated the data analysis and interpretation of results; consequently, we focused our attention on the regions we could classify confidently. Most notably, these were slightly elevated regions of $S$, alterniflora. The spectral analysis suggests the classification result was fairly accurate and it is, to some degree, in agreement with both the National Wetlands Inventory and past vegetation surveys, though both of these references are quite dated.

As mentioned earlier, the principle objective of this work is to explore the spectral variability in regards to landscape complexity, specifically at the stand or patch level. We begin this exploratory analysis by comparing several patch metrics with SFF scores in an attempt to establish trends. Our 

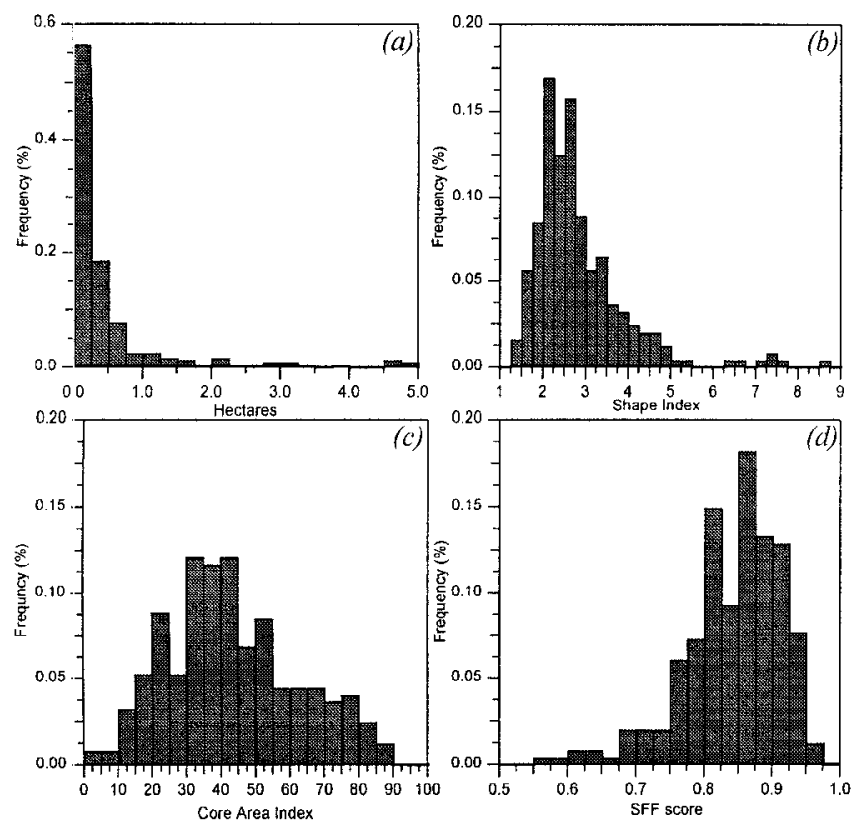

Fig. 3 Distribution for three patch metrics (a) Patch Area (b) Shape Index (c) Core Area Index and (d) SFF score

motivation was stimulated by the premise that spectral variability is reflected in landscape heterogeneity in a such away that spectral variability represents a surrogate response for plant structure and physiology. By exploring these characteristics at the patch level, it then may be possible to discern processes affecting change at the landscape level.

In regards to elevated $S$. alterniflora patches on Smith Island, SFF scores are clustered around data centers and are minimally polarized along the patch metric axis. This suggests a weak positive correlation between SFF and each metric for most of the observed relationships. Two examples are given in Fig. 4. Specifically, SFF scores exhibit an increasing trend with CAI (Fig 4a). Intuitively, this result is not surpassing since we expect regions with larger CAI to have purer stands of $S$. alterniflora (at least to some specific point), and thus a higher SFF score. Departures from this relationship could possibly be symptomatic of a physiological response such as stress or a shift in species assemblage with a latter indicating a degree of fragmentation within the patch. Fig. $4 \mathrm{~b}$ depicts SqP, shape metric. Here the relationship between SFF and the patch metric is less evident.

Clearly, the work presented here is not exhaustive and additional analysis is required in order to establish the significance of any relationship between hyperspectral measurements and landscape complexity. We anticipate additional hyperspectral data collections scheduled for this year will help clarify our analysis

\section{SUMMARY}

Initial results form this study yielded a reasonable classification mask, based on a Spectral Feature Fitting analysis, for elevated Spartina alterniflora patches, located on Smith Island, Virginia. Patch indices derived from the preceding result indicate a fair amount of complexity in regards to patch shape though core areas are large. This suggests the presence of complex shapes with fairly homogenous and extensive interiors. SFF scores also suggest a relatively uniform environment. Some trends are observed between SFF and the various patch metrics examined in this study, most notably the relationship betweeen SFF and CAI.

\section{REFERENCES}

[1] M. G. Turner, "Landscape ecology: the effect of pattern and process" Annual Rev. of Ecol. and Syst. vol. 20. pp. 171-197. 1989

[2] R. T. T Forman and M. Gordon, Landscape Ecology. John Wiles \& Sons, New York, 1986.

[3] M. G. Turner and R. H. Gardner, Quantitative Methods in Landscape Ecology, Springer-Verlag, New York, 1992.

[4] K. McGarigal and B. J. Marks. Fragstats: Spatial Analysis Program for Quantifying Landscape Structure, Version 2, Oregon State University, Corvallis, OR, 1994.

[5] K. Finkelstein, "An ephemeral inlet from the Virginia barrier Island Chain: stratigraphic sequence and Preservational potential of infilled sediments", in D. G. Aubery and L. W. Weishar, eds., Hydrodynamics and Sediment Dynamics of Tidal Inlets, Lecture Notes on Coastal and Estuarine Studies, vol. 29. Springer-Verlag, New York, 1988.

[6] B. Gao, K. B. Heidebrecht, and A. F. H. Goetz1, Atmosphere Removal Program (ATREM) version 3.1 User's GuideCenter for the Study of Earth From Space, Cooperative Institute for Research in Environmental Sciences, University of Colorado, Boulder, 1999

[7] J. A. Richards and X. Jia, Remote Sensing Digital Image Analysis-An Introduction $3^{\text {rtl }}$ ed. Springer-Verlag, NY, 1999.

[8] R. C. Frohn, Remote Sensing for Landscape Ecology: new metric indicators for monitoring, modeling, and assessment of ecosystems, Lewis Publishers, Boca Raton, FL. 1998

[9] R. N. Clark, Spectroscopy of Rocks and minerals and principles of spectroscopy in Manual of Remote Sensing 3rd ,vol.3, A. N. Rencz ed, John Wiley \& Sons, New York
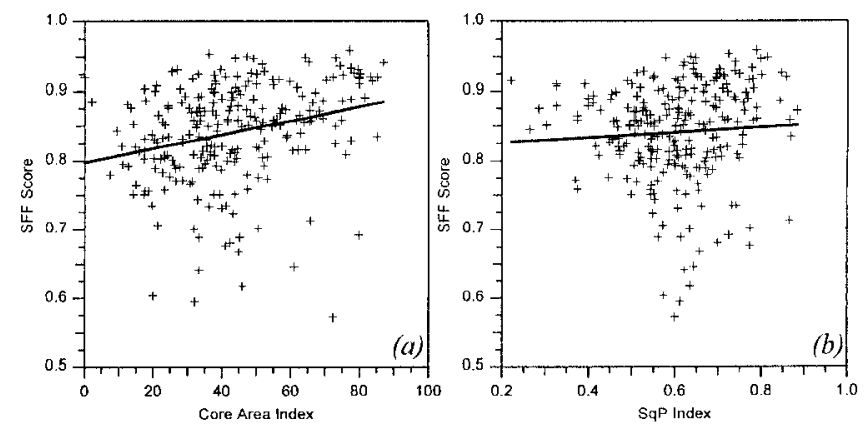

Fig. 4 Scatter plots for (a) SFF vs Core Area Index and (b) SFF vs Square Pixel metric 